\title{
Bifurcations of a Homoclinic Orbit to Saddle-Center in Reversible Systems
}

\author{
Zhiqin Qiao ${ }^{1}$ and Yancong $X_{u^{2}}$ \\ ${ }^{1}$ Department of Mathematics, North University of China, Taiyuan 030051, China \\ ${ }^{2}$ Department of Mathematics, Hangzhou Normal University, Hangzhou 310036, China
}

Correspondence should be addressed to Yancong Xu, yancongx@163.com

Received 24 July 2012; Accepted 13 October 2012

Academic Editor: Xinan Hao

Copyright (C) 2012 Z. Qiao and Y. Xu. This is an open access article distributed under the Creative Commons Attribution License, which permits unrestricted use, distribution, and reproduction in any medium, provided the original work is properly cited.

The bifurcations near a primary homoclinic orbit to a saddle-center are investigated in a 4dimensional reversible system. By establishing a new kind of local moving frame along the primary homoclinic orbit and using the Melnikov functions, the existence and nonexistence of 1-homoclinic orbit and 1-periodic orbit, including symmetric 1-homoclinic orbit and 1-periodic orbit, and their corresponding codimension 1 or codimension 3 surfaces, are obtained.

\section{Introduction}

In recent years, there are much interest in the phenomenon of homoclinics and heteroclinics in reversible dynamical systems because of their extensive applications in mechanics, fluids, and optics [1-10]. For example, Klaus and Knobloch [4] considered a homoclinic orbit to saddle-center with two-parameter families of non-Hamiltonian reversible vector fields by Lin's method. They derived the occurrence of 1-homoclinic orbits to the center manifold. Liu et al. [6] studied a singular perturbation system with action-angle variable and the unperturbed system was assumed to possess a saddle-center equilibrium in a general system without reversible or Hamiltonian structure. Mielke et al. [7] investigated bifurcations of homoclinic orbit to saddle-center in 4-dimensional reversible Hamiltonian systems. By using the Poincaré map and a special normal form, they detected the existence of $\mathrm{N}$ homoclinic orbits to the equilibrium, $N$-periodic orbits, and chaotic behavior near the primary homoclinic orbit. As for purely Hamiltonian system in $\mathbb{R}^{4}$, similar results are known from Koltsova and Lerman [2,5]. In all of these papers the underlying Hamiltonian structure was heavily considered. Especially it was used to detect multiround orbits. Note that, the Hamiltonian can make the dynamics constrict in three-dimensional manifold in a zero level 
set. In this paper, we use the method originated by Zhu [9], by constructing a local moving coordinate system and Poincaré map near the primary homoclinic orbit, the existence of transversal homoclinic orbits and periodic orbits bifurcated from the primary homoclinic orbit are obtained in a 4-dimensional reversible system. It is worth to mention that a new kind of moving coordinates is introduced firstly in our paper in order to simplify and facilitate the reversible system.

The remainder of this paper is organized as follows. Section 2 contains the assumptions for the perturbed and unperturbed system. The local coordinate moving frame, cross sections, and Poincaré map are set up in Section 3. Finally, we obtain the existence and nonexistence of 1-homoclinic orbit and 1-periodic orbit, including symmetric 1-homoclinic orbit and 1-periodic orbit, and their corresponding surfaces with different condimensions in Sections 4 and 5.

\section{The General Setup}

Consider the following system

$$
\dot{z}=f(z)+\epsilon g(z ; \mu), \quad z \in \mathbb{R}^{4}, \mu \in \mathbb{R}^{l}
$$

and the corresponding unperturbed system

$$
\dot{z}=f(z),
$$

where $0<\epsilon \ll 1, l \geq 1, f: \mathbb{R}^{4} \rightarrow \mathbb{R}^{4}$ and $g: \mathbb{R}^{4} \times \mathbb{R} \rightarrow \mathbb{R}^{4}$ are $C^{r}(r \geq 4), f(0)=g(0 ; \mu)=0$ and $\mu$ is a parameter. Also, we need the following assumptions.

(A1) System (2.1) is reversible with respect to the linear involution $R$ such that $\operatorname{dim}($ Fix $R)=2, f(R z)+R f(z)=g(R z ; \mu)+R g(z ; \mu)=0$ for all $z \in \mathbb{R}^{4}$ and $\mu \in R^{l}$.

Note that, throughout the paper, we will denote $R$-symmetric orbit as symmetric orbit.

(A2) The origin $O$ is a saddle-center equilibrium of (2.2), $O \in$ Fix $R$. More precisely, the Jacobian matrix $A \triangleq D f(0)$ has a pair of purely imaginary eigenvalues $\pm i \omega$ and two nonzero real eigenvalues, that is, $\sigma(A)=\{ \pm i \omega, \pm \lambda\}$ with $\omega, \lambda>0$.

(A3) System (2.2) has a symmetric homoclinic orbit $\Gamma=\{z=r(t): t \in \mathbb{R}\}$, where $r( \pm \infty)=O$.

Note that, $O$ is a symmetric equilibrium, and the eigenvalues of the Jacobian matrix $A$ are symmetric with respect to the imaginary axis. Thus, assumption (A2) describes a scenario that is structurally stable. Furthermore, the saddle-center $O$ has one-dimensional stable manifold $W_{\epsilon, \mu}^{s}(O)$ and one-dimensional unstable manifolds $W_{\epsilon, \mu}^{u}(O)$ (abbr. $W^{s}$ and $W^{u}$ as $\epsilon=0$ ), and a two-dimensional center manifold $W_{\epsilon, \mu}^{c}(O)\left(\right.$ abbr. $W^{c}$ as $\epsilon=0$ ) for $\epsilon$ close to 0 . All of them are $C^{r}$. Confined to $W_{\epsilon, \mu}^{c}(O), O$ is a center. The reversibility implies that $W_{\epsilon, \mu}^{u}(O)=R W_{\epsilon, \mu}^{s}(O)$ and $W_{\epsilon, \mu}^{c}(O)=R W_{\epsilon, \mu}^{c}(O)$, and hence, the homoclinic orbit $\Gamma$ is symmetric, that is, $R \Gamma=\Gamma$.

\section{Local Moving Frame and Poincaré Map}

Suppose the neighborhood $U_{0}$ of $O$ is small enough, we can firstly straighten the centerstable manifold, the center-unstable manifold, subsequently, then the stable manifold and 
the unstable manifold in $U_{0}$ by using the method introduced in Zhu [9]. According to the invariance and symmetry of these manifolds, we can deduce that system (2.1) has the following form in $U_{0}$ :

$$
\begin{gathered}
\dot{x}=x(\lambda(\epsilon, \mu)+O(1)), \\
\dot{y}=y(-\lambda(\epsilon, \mu)+O(1)), \\
\dot{u}=v(\omega(\epsilon, \mu)+O(1))+u(O(x)+O(y)+O(u))+O(x y), \\
\dot{v}=u(-\omega(\epsilon, \mu)+O(1))-v(O(x)+O(y)+O(v))-O(x y),
\end{gathered}
$$

where $\lambda(0, \mu)=\lambda, \omega(0, \mu)=\omega, O(1)=O(x)+O(y)+O(u)+O(v)$, the system is $C^{r-2}$, and the corresponding involution acts as $R(x, y, u, v)=(y, x, v, u)$.

In fact, by a linear transformation, system (2.1) takes the form in a small neighborhood of $U_{0}$ as follows:

$$
\begin{gathered}
\dot{x}=\lambda(\epsilon, \mu) x+O(2) \\
\dot{y}=-\lambda(\epsilon, \mu) y+O(2) \\
\dot{u}=\omega(\epsilon, \mu) v+O(2) \\
\dot{v}=-\omega(\epsilon, \mu) u+O(2)
\end{gathered}
$$

and $R(x, y, u, v)=(y, x, v, u)$. By the invariant manifold theorem, we know that there exist a local $C^{r}$ center-stable manifold $W_{\epsilon, \mu}^{c s}=\left\{z=(x, y, u, v): x=x_{\epsilon, \mu}^{c s}(y, u, v), x_{\epsilon, \mu}^{c s}(0,0,0)=\right.$ $\left.0, D x_{\epsilon, \mu}^{c s}(0,0,0)=0, z \in U_{0}\right\}$, a local $C^{r}$ center-unstable manifold $W_{\epsilon, \mu}^{c u}=\{z=(x, y, u, v): y=$ $\left.y_{\epsilon, \mu}^{c u}(x, u, v), y_{\epsilon, \mu}^{c u}(0,0,0)=0, D y_{\epsilon, \mu}^{c u}(0,0,0)=0, z \in U_{0}\right\}$ and $R W_{\epsilon, \mu}^{c s}=W_{\epsilon, \mu}^{c u}$.

By the straightening coordinate transformation which is similar to that of $[1,6,9]$, now we straighten the local manifolds $W_{\varepsilon, \mu}^{c s}$ and $W_{\varepsilon, \mu}^{c u}$, such that $W_{\epsilon, \mu}^{c s}=\left\{z \in U_{0}: x=0\right\}, W_{\epsilon, \mu}^{c u}=$ $\left\{z \in U_{0}: y=0\right\}$.

Notice that the invariance of $W_{\epsilon, \mu}^{c s}$ and $W_{\epsilon, \mu}^{c u}$ implies the local invariance of $\left\{z \in U_{0}\right.$ : $x=0\}$ and $\left\{z \in U_{0}: y=0\right\}$, respectively, which produces that, in $U_{0}$,

$$
\begin{gathered}
\dot{x}=x(\lambda(\epsilon, \mu)+O(1)), \\
\dot{y}=y(-\lambda(\epsilon, \mu)+O(1)) .
\end{gathered}
$$

Now the system is $C^{r-1}$ and still reversible. By using a similar procedure to straighten the local $C^{r-1}$ stable manifold $W_{\epsilon, \mu}^{s}$ and unstable manifold $W_{\epsilon, \mu}^{u}$, and the invariance and symmetry of these two local manifolds (that means the transformation is also symmetric), we get system (3.1). Clearly, corresponding to system (3.1), the center manifold $W_{\epsilon, \mu}^{c}$ is locally in the $u-v$ plane, and the stable manifold $W_{\epsilon, \mu}^{s}$ (resp., unstable manifold $W_{\epsilon, \mu}^{u}$ ) is locally the $y$-axis (resp., $x$-axis) when they are confined in $U_{0}$.

Define $r(-T)=(\delta, 0,0,0)^{*}, r(T)=(0, \delta, 0,0)^{*}$ for $T \gg 1$, where $\delta>0$ is small enough such that $\left\{(x, y, u, v)^{*}:|x|,|y|,\left|u^{2}+v^{2}\right|^{1 / 2}<2 \delta\right\} \subset U_{0}$. 
Consider the linear variational system

$$
\dot{z}=D f(r(t)) z
$$

and its adjoint system

$$
\dot{\phi}=-(D f(r(t)))^{*} \phi
$$

Based on the invariance and symmetry of manifolds $W^{c s}$ and $W^{c u}$, it is easy to know that system (3.4) has a fundamental solution matrix $Z(t)=\left(z_{1}(t), z_{2}(t), z_{3}(t), z_{4}(t)\right)$ satisfying $z_{1}(t) \in\left(T_{r(t)} W^{c u}\right)^{c}, z_{2}(t)=-\dot{r}(t) /|\dot{r}(T)| \in T_{r(t)}\left(W^{s} \cap W^{u}\right), z_{3}(t), z_{4}(t) \in T_{r(t)} W^{c u}$ and

$$
Z(-T)=\left(\begin{array}{cccc}
0 & -1 & 0 & 0 \\
1 & 0 & 0 & 0 \\
0 & 0 & 1 & 0 \\
0 & 0 & 0 & 1
\end{array}\right), \quad Z(T)=\left(\begin{array}{cccc}
1 & 0 & 0 & 0 \\
0 & 1 & 0 & 0 \\
0 & 0 & 0 & 1 \\
0 & 0 & -1 & 0
\end{array}\right)
$$

Actually, in the resulting coordinates, $W^{s} \cap U_{0}$ and $W^{u} \cap U_{0}$ are $y$-axis and $x$-axis, respectively, combining with the symmetry, it follows that

$$
z_{2}(T)=(0,1,0,0)^{*}, \quad z_{2}(-T)=(-1,0,0,0)^{*}
$$

On the other hand, in a small tubular neighborhood of the homoclinic loop $\Gamma$, the center-unstable manifold $W^{c u}$ (resp., center-stable manifold $W^{c s}$ ) can be foliated into a family of leaves, each is a 2-dimensional surface and asymptotic to $W^{c}$ as the base point $z=r(t) \in \Gamma$ tends to $O$ as $t \rightarrow-\infty$ (resp., $+\infty$ ). Notice that the limit of the linearization (3.4) of system (2.2) with respect to $\Gamma$ as $t \rightarrow \pm \infty$ is

$$
\dot{x}=\lambda x, \quad \dot{y}=-\lambda y, \quad \dot{u}=\omega v, \quad \dot{v}=-\omega u .
$$

When confined on $W^{c}$, it becomes the following subsystem

$$
\dot{u}=\omega v, \quad \dot{v}=-\omega u,
$$

which is reversible with the involution $(u, v) \rightarrow(v, u)$. Obviously, for any $a>0$ and $t_{0} \in R$, $u=u(t)=a \sin \omega\left(t-t_{0}\right), v=v(t)=a \cos \omega\left(t-t_{0}\right)$ is a solution of (3.9), which defines a closed orbit $\widehat{\Gamma}$ on $W^{c}$. More precisely, its tangent vectors $u_{1}(t)=\dot{u}(t)=a \omega \cos \omega\left(t-t_{0}\right)$, $v_{1}(t)=\dot{v}(t)=-a \omega \sin \omega\left(t-t_{0}\right)$ and its normal vectors $u_{2}(t)=-\dot{v}(t)=a \omega \sin \omega\left(t-t_{0}\right)$, $v_{2}(t)=\dot{u}(t)=a \omega \cos \omega\left(t-t_{0}\right)$ are the solutions of (3.9). Choose some appropriate $a$ and $t_{0}$ such that $a w=1, \omega\left(-T-t_{0}\right)=2 k \pi$ for some $k \in Z$, then $(u(-T), v(-T))=(0, a)=$ $\left(0, w^{-1}\right), \bar{z}_{3}(-T)=\left(u_{1}(-T), v_{1}(-T)\right)=(1,0), \bar{z}_{4}(-T)=\left(u_{2}(-T), v_{2}(-T)\right)=(0,1)$. Based on the reversibility, we have $(u(T), v(T))=(a, 0)=\left(w^{-1}, 0\right), \bar{z}_{3}(T)=\left(u_{1}(T), v_{1}(T)\right)=$ $(0,-1), \bar{z}_{4}(T)=\left(u_{2}(T), v_{2}(T)\right)=(1,0)$ (see Figure 1 for details). Thus, if we take solutions $z_{3}(t)$ and $z_{4}(t)$ in $T_{r(t)} W^{c u}$ satisfying $z_{3}(-T)=(0,0,1,0)^{*}, z_{4}(-T)=(0,0,0,1)^{*}$, then, restricted 


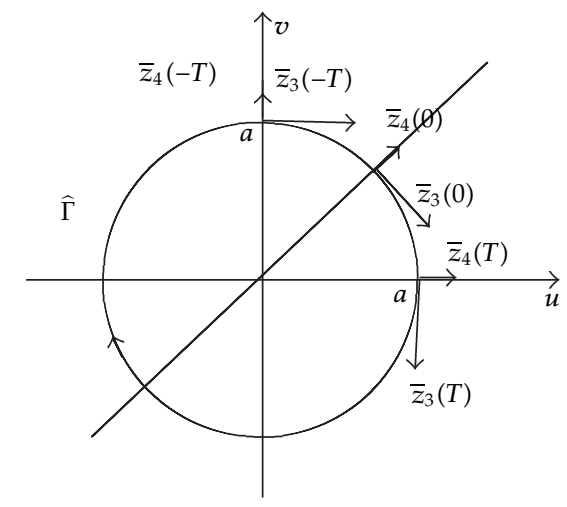

Figure 1: The geometry of vectors on the center manifold.

to the $u-v$ plane, $z_{3}(-T)$ is the unit tangent direction of the closed orbit $\widehat{\Gamma}$ at $(u, v)=(0, a)=$ $\left(0, \omega^{-1}\right)$. In addition, the restriction of $z_{4}(-T)$ is its unit exterior normal direction at the same point. By the reversibility, it is easy to obtain $z_{3}(T)=(0,0,0,-1)^{*}, z_{4}(T)=(0,0,1,0)^{*}$.

Finally, if we choose a solution $z_{1}(t) \in\left(T_{r(t)} W^{c u}\right)^{c}$ with $z_{1}(-T)=(0,1,0,0)^{*}$, then the symmetry says that $z_{1}(T)=(1,0,0,0)^{*}$.

Therefore, we have demonstrated the existence of the fundamental matrix $Z(t)$ with the specified properties.

Remark 3.1. In the following, we will regard $\left(z_{1}(t), z_{2}(t), z_{3}(t), z_{4}(t)\right)$ as a moving coordinate in a small tubular neighborhood of $\Gamma$. This new kind of moving frame is firstly introduced for the homoclinic orbit to a saddle-center, which is the extension of the corresponding coordinates built in $[1,6,9]$ for the homoclinic orbit to a saddle. The explicit advantage is that, these coordinate vectors inherit and exhibit the geometrical and dynamical properties of those invariant manifolds. As mentioned above, they will greatly simplify the original reversible system.

Let $\Sigma^{u}=\left\{(x, y, u, v)|x=\delta| y,|,| u^{2}+\left.v^{2}\right|^{1 / 2} \leq \delta / 2\right\} \subset U_{0}$ and $\Sigma^{s}=\{(x, y, u, v) \mid y=$ $\left.\delta,|x|,\left|u^{2}+v^{2}\right|^{1 / 2} \leq \delta / 2\right\} \subset U_{0}$ be the cross sections of $\Gamma$ at $t=-T$ and $t=T$, respectively.

Now we turn to seek the new coordinates of $q_{0} \in \Sigma^{u}$ and $q_{1} \in \Sigma^{s}$ (see Figure 2 for details) under the transformation $z(t)=r(t)+Z(t) \cdot N$, where $N=\left(n_{1}, 0, n_{3}, n_{4}\right)^{*}$. Take

$$
\begin{gathered}
q_{0} \triangleq\left(x_{0}, y_{0}, u_{0}, v_{0}\right)=r(-T)+Z(-T) \cdot\left(n_{1}^{0}, 0, n_{3}^{0}, n_{4}^{0}\right)^{*}, \\
q_{1} \triangleq\left(x_{1}, y_{1}, u_{1}, v_{1}\right)=r(T)+Z(T) \cdot\left(n_{1}^{1}, 0, n_{3}^{1}, n_{4}^{1}\right)^{*},
\end{gathered}
$$

which are solved by

$$
\begin{aligned}
& x_{0}=\delta, \quad n_{1}^{0}=y_{0}, \quad n_{3}^{0}=u_{0}, \quad n_{4}^{0}=v_{0} ; \\
& y_{1}=\delta, \quad n_{1}^{1}=x_{1}, \quad n_{3}^{1}=-v_{1}, \quad n_{4}^{1}=u_{1} \text {. }
\end{aligned}
$$




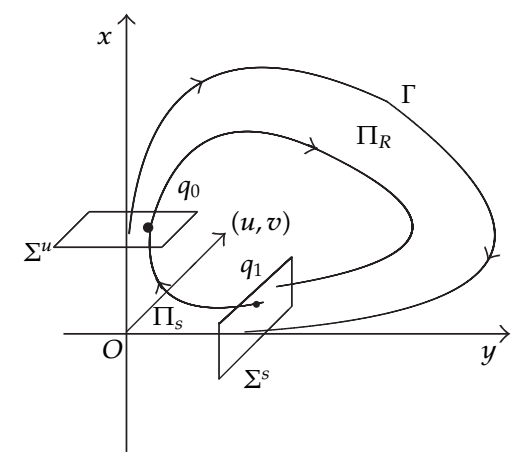

Figure 2: Setup of cross sections and Poincaré map.

Putting the transformation $z(t)=r(t)+Z(t) \cdot N$ into (2.1), we get

$$
\begin{aligned}
\dot{r}(t)+\dot{Z}(t) \cdot N+Z(t) \cdot \dot{N} & =f(r(t)+Z(t) \cdot N)+\epsilon g(r(t)+Z(t) \cdot N, \mu) \\
& =f(r(t))+D f(r(t)) Z(t) \cdot N+\epsilon g(r(t), \mu)+O\left((\epsilon+N)^{2}\right) .
\end{aligned}
$$

That implies $Z(t) \cdot \dot{N}=\epsilon g(r(t), \mu)+O\left((\epsilon+N)^{2}\right)$, which is $C^{r-3}$. Then multiplying the resulting equation by $Z^{-1}(t)=\left(\phi_{1}^{*}, \phi_{2}^{*}, \phi_{3}^{*}, \phi_{4}^{*}\right)^{*}$ and integrating it on both sides from $-T$ to $T$, we have the regular map $\Pi_{R}: \Sigma^{u} \rightarrow \Sigma^{s}$ :

$$
n_{i}(T)=n_{i}(-T)+\epsilon M_{i}(T, \mu)+O\left((\epsilon+N(-T))^{2}\right), \quad i=1,3,4
$$

where $M_{i}(T, \mu)=\int_{-T}^{T} \phi_{i}^{*} g_{i}(r(t), \mu) d t$ are the Melnikov functions.

For conciseness, we will denote $\lambda(\epsilon, \mu) \triangleq \lambda, \omega(\epsilon, \mu) \triangleq \omega$. Now we consider the local map $\Pi_{S}$ induced by the flow (3.1) in $U_{0}$, where

$$
\Pi_{S}: \Sigma^{s} \longrightarrow \Sigma^{u}, \quad q_{1}=\left(n_{1}^{1}, 0, n_{3}^{1}, n_{4}^{1}\right) \longrightarrow q_{0}=\left(n_{1}^{0}, 0, n_{3}^{0}, n_{4}^{0}\right)
$$

Let $\tau$ be the flying time from $q_{1}$ to $q_{0}$, by variation of constants formula, we can get the following expression:

$$
\begin{gathered}
x_{1}=x(T)=x_{0} e^{-\lambda \tau}\left(1+\rho_{1}\right), \\
y_{0}=y(T+\tau)=y_{1} e^{-\lambda \tau}\left(1+\rho_{2}\right), \\
u_{0}=u(T+\tau)=\left[u_{1} \cos (\omega \tau)+v_{1} \sin (\omega \tau)\right]\left(1+\rho_{3}\right)+\rho_{5}, \\
v_{0}=v(T+\tau)=\left[-u_{1} \sin (\omega \tau)+v_{1} \cos (\omega \tau)\right]\left(1+\rho_{4}\right)+\rho_{6},
\end{gathered}
$$

where $\rho_{i}=\rho_{i}\left(x_{0}, y_{1}, u_{1}, v_{1}, \tau, \epsilon, \mu\right), \rho_{i} \in C^{0}, i=1, \ldots, 6$, and $\rho_{i}=O(\delta)$ for $i=1,2,3,4, \rho_{j}=$ $O\left(\delta^{2} e^{-\lambda \tau}\right)$ for $j=5,6$. 
Denote $s=e^{-\lambda \tau}$, then we get $\Pi_{S}: \Sigma^{s} \rightarrow \Sigma^{u}$ defined by

$$
\begin{gathered}
n_{1}^{0}=\delta s\left(1+\rho_{2}\right), \\
n_{3}^{0}=\left[n_{4}^{1} \cos \left(\frac{\omega}{\lambda} \log s\right)+n_{3}^{1} \sin \left(\frac{\omega}{\lambda} \log s\right)\right]\left(1+\rho_{3}\right)+\rho_{5}, \\
n_{4}^{0}=\left[n_{4}^{1} \sin \left(\frac{\omega}{\lambda} \log s\right)-n_{3}^{1} \cos \left(\frac{\omega}{\lambda} \log s\right)\right]\left(1+\rho_{4}\right)+\rho_{6} .
\end{gathered}
$$

Combining the maps (3.13) and (3.16), we obtain the Poincaré map $\Pi=\Pi_{R} \circ \Pi_{S}$ : $\Sigma^{s} \rightarrow \Sigma^{s}$ defined as

$$
\begin{gathered}
n_{1}(T)=\delta s\left(1+\rho_{2}\right)+\epsilon M_{1}(T, \mu)+\text { h.o.t., } \\
n_{3}(T)=\left[n_{4}^{1} \cos \left(\frac{\omega}{\lambda} \log s\right)+n_{3}^{1} \sin \left(\frac{\omega}{\lambda} \log s\right)\right]\left(1+\rho_{3}\right)+\epsilon M_{3}(T, \mu)+\rho_{5}+\text { h.o.t., } \\
n_{4}(T)=\left[n_{4}^{1} \sin \left(\frac{\omega}{\lambda} \log s\right)-n_{3}^{1} \cos \left(\frac{\omega}{\lambda} \log s\right)\right]\left(1+\rho_{4}\right)+\epsilon M_{4}(T, \mu)+\rho_{6}+\text { h. o.t. }
\end{gathered}
$$

\section{Existence of 1-Homoclinic Orbit and 1-Periodic Orbit}

Let $G\left(q_{1}\right) \triangleq \Pi\left(q_{1}\right)-q_{1}$ be the displacement function. Based on (3.11), (3.17), and the new coordinate of $q_{1}=\left(n_{1}^{1}, 0, n_{3}^{1}, n_{4}^{1}\right)$, we see the small zero point $\left(s, u_{1}, v_{1}\right)$ of $G$ will satisfy the following equations:

$$
\begin{gathered}
\delta s\left(\rho_{2}-\rho_{1}\right)+\epsilon M_{1}(T, \mu)+O\left(|s, \epsilon|^{2}\right)=0, \\
v_{1}+\left[u_{1} \cos \left(\frac{\omega}{\lambda} \log s\right)-v_{1} \sin \left(\frac{\omega}{\lambda} \log s\right)\right]\left(1+\rho_{3}\right)+\epsilon M_{3}(T, \mu)+\rho_{5}+\text { h.o.t. }=0, \\
u_{1}-\left[u_{1} \sin \left(\frac{\omega}{\lambda} \log s\right)+v_{1} \cos \left(\frac{\omega}{\lambda} \log s\right)\right]\left(1+\rho_{4}\right)-\epsilon M_{4}(T, \mu)-\rho_{6}+\text { h.o.t. }=0 .
\end{gathered}
$$

Due to the coordinate transformations introduced in $U_{0}$ at the beginning of Section 3 , the unstable manifold and the stable manifold are locally $x$-axis and $y$-axis, respectively, so it is evident that, near $\Gamma$, system (2.1) has a symmetric 1-homoclinic orbit to $O$ if and only if (4.1) have a solution $\left(s, u_{1}, v_{1}\right)$ with $s=u_{1}=v_{1}=0$, and system (2.1) has an 1-homoclinic orbit to a periodic orbit on the center manifold and an 1-periodic orbit if and only if (4.1) have a solution $\left(s, u_{1}, v_{1}\right)$ with $s=0, u_{1}^{2}+v_{1}^{2}>0$ and $s>0, u_{1}^{2}+v_{1}^{2} \geq 0$, respectively. Clearly, system (4.1) is $C^{1}$ in $\left(s, u_{1}, v_{1}\right)$ as $0 \leq s \ll 1, u_{1}^{2}+v_{1}^{2} \ll 1$, and $C^{3}$ in $(\epsilon, \mu)$, and has a solution $s=u_{1}=v_{1}=0$ as $\epsilon=0$, thus we can rescale $s=\epsilon^{2} \bar{s}_{,} u_{1}=\epsilon^{2} u, v_{1}=\epsilon^{2} v$, such that system (4.1) is reformulated as

$$
\begin{aligned}
& M_{1}(T, \mu)+O(\epsilon)=0, \\
& M_{3}(T, \mu)+O(\epsilon)=0, \\
& M_{4}(T, \mu)+O(\epsilon)=0,
\end{aligned}
$$

where $O(\epsilon)$ depends on $\epsilon, \bar{s}, u, v$ and $\mu$. 
Now the following results are verified directly by the implicit function theorem.

Theorem 4.1. Assume (A1)-(A3) are satisfied, $M_{1}\left(T, \mu_{0}\right)=M_{3}\left(T, \mu_{0}\right)=M_{4}\left(T, \mu_{0}\right)=0$ and $\operatorname{Rank}\left(\partial\left(M_{1}, M_{3}, M_{4}\right) / \partial \mu\right)\left(T, \mu_{0}\right)=3$ for $l \geq 3$ and $\mu_{0} \in \mathbb{R}^{l}$, then, for any given $r_{0}>0, \bar{s}_{0}>0$ and $\epsilon_{0}>0$ small enough, there exist a single-parameter family of codimension 3 surface $\Sigma_{\epsilon}$ and a four-parameter family of codimension 3 surface $\Sigma_{\text {euvs }}$ near $\mu_{0}$ such that $\Sigma_{0}=\Sigma_{0 u v \bar{s}}=\left\{\mu \mid \mu=\mu_{0}\right\}$, $\lim _{\bar{s} \rightarrow 0} \Sigma_{\epsilon u v \bar{s}}=\Sigma_{\epsilon u v 0}$ for $0<\epsilon<\epsilon_{0}, 0 \leq u^{2}+v^{2}<r_{0}^{2}, 0<\bar{s}<\bar{s}_{0}$, and system (2.1) has a symmetric 1-homoclinic orbit to $O$ as $\mu \in \Sigma_{\epsilon}$, a 1-periodic orbit with approximate period $2 T-\lambda^{-1} \log \epsilon^{2} \bar{s}$ as $\mu \in \Sigma_{\text {euvs }}$, and a 1-homoclinic orbit to a periodic orbit on the center manifold with $u_{1}^{2}(t)+v_{1}^{2}(t)=$ $\epsilon^{4}\left(u_{0}^{2}+v_{0}^{2}\right)$ as $\mu \in \Sigma_{\epsilon u_{0} v_{0} 0}, \Sigma_{\epsilon u_{0} v_{0} 0}-\left\{\mu_{0}\right\} \neq \emptyset, 0<u_{0}^{2}+v_{0}^{2}<r_{0}^{2}$. Moreover, these codimension 3 surfaces are either coincident, or tangent to each other at $\mu=\mu_{0}$, and they have a common 3-dimensional normal space spanned by $M_{1 \mu}\left(T, \mu_{0}\right), M_{3 \mu}\left(T, \mu_{0}\right)$, and $M_{4 \mu}\left(T, \mu_{0}\right)$ as $l>3$.

In order to study the existence of the 1-periodic orbit bifurcation surface with lower codimension, we reconsider (4.1). Let $s=e^{-(\lambda / \omega)(2 k \pi-\alpha)}, \alpha \in[-(\pi / 2),(3 \pi / 2))$ and $H$ be the vector defined by the left hand side of the last two equations of (4.1), then, by using

$$
\begin{aligned}
W \triangleq \operatorname{det}\left[\left.\frac{\partial H}{\partial\left(u_{1}, v_{1}\right)}\right|_{\substack{u_{1}=v_{1}=\epsilon=0 \\
k=\infty}}\right] \\
=-\left.\left[2+\rho_{3}+\rho_{4}+\rho_{3} \rho_{4}-\left(2+\rho_{3}+\rho_{4}\right) \sin \alpha\right]\right|_{\substack{u_{1}=v_{1}=\epsilon=0 \\
k=\infty}},
\end{aligned}
$$

it follows that, for $\delta>0$ so small that $\left|\rho_{3}+\rho_{4}\right|<0.1,\left|\rho_{3}+\rho_{4}+\rho_{3} \rho_{4}\right|<0.1$, we have $W \neq 0$ for $\alpha \in[-(\pi / 4),(5 \pi / 4)]$. Therefore, there is a unique pair $\left(u_{1}, v_{1}\right)$ which solves the last two equations as $k>-(\omega / \lambda \pi) \log \epsilon$ and $\epsilon>0$ is small enough.

Substituting this solution $\left(u_{1}, v_{1}\right)$ into the first equation of (4.1), and using $s=O\left(\epsilon^{2}\right)$ for $k>-(\omega / \lambda \pi) \log \epsilon$, it produces

$$
M_{1}(T, \mu)+O(\epsilon)=0
$$

where the term $O(\epsilon)$ depends on $\epsilon, k, \alpha$ and $\mu$.

At this stage, we have actually demonstrated the following existence theorem.

Theorem 4.2. Suppose $(A 1)-(A 3)$ are satisfied, $M_{1}\left(T, \mu_{0}\right)=0,\left(\partial M_{1} / \partial \mu\right)\left(T, \mu_{0}\right) \neq 0$ as $l \geq 1$ and $\mu_{0} \in \mathbb{R}^{l}$, then for $\epsilon_{0}>0, \delta>0$ small enough, $0<\epsilon<\epsilon_{0}, k>-(\omega / \lambda \pi) \log \epsilon$ and $\alpha \in[-(\pi / 4),(5 \pi / 4)]$, there exists a 3-parameter (resp., 2-parameter) family of codimension 1 surface $\Sigma_{\epsilon k \alpha}\left(\right.$ resp., $\left.\Sigma_{\epsilon \alpha}=\Sigma_{\epsilon \infty \alpha}\right)$ near $\mu_{0}$ satisfying $\Sigma_{0 \infty \alpha}=\left\{\mu_{0}\right\}$, such that system (2.1) has one 1-periodic orbit $L(\epsilon, k, \alpha)$ (resp., 1-homoclinic orbit $L(\epsilon, \infty, \alpha)$ ) near $\Gamma$ as $\mu \in \Sigma_{\epsilon k \alpha}\left(r e s p ., \mu \in \Sigma_{\epsilon \alpha}\right.$ ), and $L(\epsilon, k, \alpha)$ has an approximate period $2 T+((2 k \pi-\alpha) / \omega)$. In addition, if $M_{1}(T, \mu) \neq 0$ for any $\mu \in \mathbb{R}^{l}$, then system (2.1) has not any 1-periodic orbit near $\Gamma$.

Remark 4.3. When confined in a neighborhood of the local center-manifold, the $(u, v)$ coordinates of 1-periodic or 1-homoclinic orbit guaranteed by Theorem 4.1 as $\mu \in \Sigma_{\text {euv } \bar{s}}$ or $\mu \in$ $\Sigma_{\epsilon u_{0} v_{0} 0}$ satisfying $u_{1}^{2}+v_{1}^{2}=O\left(\epsilon^{4}\right)$, whereas, these coordinates of the corresponding orbits given in Theorem 4.2 as $\mu \in \Sigma_{\epsilon k \alpha}$ or $\mu \in \Sigma_{\epsilon \alpha}$ have the scale $O\left(\epsilon^{2}\right)$ in case $M_{4}^{2}\left(T, \mu_{0}\right)+M_{3}^{2}\left(T, \mu_{0}\right) \neq 0$ and $k>-(\omega / \lambda \pi) \log \epsilon$. 


\section{Existence of Symmetric 1-Periodic Orbit}

In this section, we turn to seek the existence of symmetric 1-periodic orbit. Note that, a 1periodic orbit $L$ near $\Gamma$ is symmetric if and only if $R\left(L \cap \Sigma^{s}\right)=L \cap \Sigma^{u}$, equivalently $L \cap \Sigma^{s}=$ $\left(x_{1}, \delta, u_{1}, v_{1}\right)$ and $L \cap \Sigma^{u}=\left(\delta, y_{0}, u_{0}, v_{0}\right)$ should satisfy

$$
x_{1}=y_{0}, \quad u_{1}=v_{0}, \quad v_{1}=u_{0} .
$$

Due to (3.15), it is equivalent to

$$
\begin{gathered}
\rho_{1}-\rho_{2}=0, \\
u_{1}-\left[u_{1} \sin \left(\frac{\omega}{\lambda} \log s\right)+v_{1} \cos \left(\frac{\omega}{\lambda} \log s\right)\right]\left(1+\rho_{4}\right)-\rho_{6}=0, \\
v_{1}-\left[u_{1} \cos \left(\frac{\omega}{\lambda} \log s\right)-v_{1} \sin \left(\frac{\omega}{\lambda} \log s\right)\right]\left(1+\rho_{3}\right)-\rho_{5}=0 .
\end{gathered}
$$

Using (5.2) and rescaling $u_{1}=\epsilon u, v_{1}=\epsilon v, s=e^{-(\lambda / \omega)(4 k \pi+2 \theta)}=O\left(\epsilon^{2}\right)$ for $k>-(\omega / 2 \lambda \pi) \log \epsilon$, $\theta \in[0,2 \pi)$, system $(4.1)$ reads as

$$
\begin{gathered}
M_{1}(T, \mu)+O(\epsilon)=0, \\
2 v+M_{3}(T, \mu)+O(\epsilon)=0, \\
M_{4}(T, \mu)+O(\epsilon)=0 .
\end{gathered}
$$

Solving the second equation of (5.3), we have

$$
v=-\frac{1}{2} M_{3}(T, \mu)+O(\epsilon) .
$$

On the other hand, $L \cap \Sigma^{S}$ and $L \cap \Sigma^{u}$ are symmetric if and only if their middle point $(x(T+(1 / 2) \tau), y(T+(1 / 2) \tau), u(T+(1 / 2) \tau), v(T+(1 / 2) \tau))$ lies on the plane Fix $(R)$, that is, (5.1) is equivalent to

$$
x\left(T+\frac{1}{2} \tau\right)=y\left(T+\frac{1}{2} \tau\right), \quad u\left(T+\frac{1}{2} \tau\right)=v\left(T+\frac{1}{2} \tau\right),
$$


where

$$
\begin{gathered}
x\left(T+\frac{1}{2} \tau\right)=\delta \sqrt{s}\left(1+\rho_{11}\right) \\
y\left(T+\frac{1}{2} \tau\right)=\delta \sqrt{s}\left(1+\rho_{21}\right) \\
u\left(T+\frac{1}{2} \tau\right)=\left[u_{1} \cos \left(\frac{\omega \tau}{2}\right)+v_{1} \sin \left(\frac{\omega \tau}{2}\right)\right]\left(1+\rho_{31}\right)+\rho_{51} \\
v\left(T+\frac{1}{2} \tau\right)=\left[-u_{1} \sin \left(\frac{\omega \tau}{2}\right)+v_{1} \cos \left(\frac{\omega \tau}{2}\right)\right]\left(1+\rho_{41}\right)+\rho_{61}
\end{gathered}
$$

and $\rho_{i 1}=\rho_{i 1}\left(x_{1}, y_{1}, u_{1}, v_{1}, \tau, \epsilon, \mu\right)$ for $i=1, \ldots, 6, \rho_{i 1}=O\left(x_{1} / \sqrt{s}\right)+O_{i}\left(y_{1}\right)+O\left(\sqrt{u_{1}^{2}+v_{1}^{2}}\right)=$ $O(\delta), O_{i}\left(y_{1}\right)=O\left(y_{1}\right)$ for $i=1, \ldots, 4, \rho_{j 1}=O\left(x_{1} y_{1}\right)=O\left(\delta^{2} s\right)$ for $j=5,6$.

Explicitly, (5.5) can be formulated as

$$
\begin{gathered}
\rho_{11}-\rho_{21}=0 \\
u_{1}\left[\cos \left(\frac{\omega \tau}{2}\right)+\sin \left(\frac{\omega \tau}{2}\right)\right]-v_{1}\left[\cos \left(\frac{\omega \tau}{2}\right)-\sin \left(\frac{\omega \tau}{2}\right)\right]+O(\delta)+O\left(\delta^{2} s\right)=0 .
\end{gathered}
$$

Due to $(5.4)$ and $(1 / 2) \omega \tau=2 k \pi+\theta, \theta \in[0,2 \pi)$, we can uniquely solve the second equation in (5.7) by

$$
u_{1}=-\frac{\epsilon(\cos \theta-\sin \theta)}{2(\cos \theta+\sin \theta)} M_{3}(T, \mu)(1+O(\delta))+O\left(\epsilon^{2}\right)
$$

as $\theta \neq(3 / 4) \pi,(7 / 4) \pi$, and $\delta, \epsilon$ small enough.

Up to now, there are three equations

$$
\begin{gathered}
\rho_{11}-\rho_{21}=0, \\
M_{1}(T, \mu)+O(\epsilon)=0, \\
M_{4}(T, \mu)+O(\epsilon)=0
\end{gathered}
$$

left which should be fulfilled, where $v_{1}=\epsilon v$ and $u_{1}$ are given by (5.4) and (5.8).

Applying the implicit function theorem to (5.9), where $O(\epsilon)$ depends on $\epsilon, \theta, k$ and $\mu$, we derive the following result.

Theorem 5.1. Suppose that (A1)-(A3) hold, $M_{1}\left(T, \mu_{0}\right)=0, M_{4}\left(T, \mu_{0}\right)=0$ and

$$
\operatorname{Rank}\left\{\left.\frac{\partial\left(\rho_{11}-\rho_{21}\right)}{\partial \mu}\right|_{\substack{\epsilon=0, \mu=\mu_{0} \\ k=\infty}}, \frac{\partial\left(M_{1}, M_{4}\right)}{\partial \mu}\left(T, \mu_{0}\right)\right\}=3
$$


as $\mu_{0} \in \mathbb{R}^{l}(l \geq 3)$, there exist $\epsilon_{0}>0, \theta_{0}>0$ small enough, and a 3-parameter family of codimension 3 surface $\Sigma_{e \theta k}$ near $\mu_{0}$, such that, system (2.1) has a symmetric 1-periodic orbit $L$ near $\Gamma$ with approximate period $2 T+(1 / \omega)(4 k \pi+2 \theta)$ as $\mu \in \Sigma_{\epsilon \theta k}$ for $\epsilon, \theta$, $k$ satisfying $0<\epsilon<\epsilon_{0}$, $\theta \in\left[0,(3 / 4) \pi-\theta_{0}\right) \cup\left((3 / 4) \pi+\theta_{0},(7 / 4) \pi-\theta_{0}\right) \cup\left((7 / 4) \pi+\theta_{0}, 2 \pi\right), k>-(\omega / 2 \lambda \pi) \log \epsilon$. Moreover, if there is no $\mu_{0} \in \mathbb{R}^{l}$ satisfying

$$
M_{1}^{2}\left(T, \mu_{0}\right)+M_{4}^{2}\left(T, \mu_{0}\right)+\left.\left(\rho_{11}-\rho_{21}\right)^{2}\right|_{\substack{\epsilon=0, \mu=\mu_{0} \\ k=\infty}}=0,
$$

then system (2.1) has no symmetric 1-periodic orbit in the small neighborhood of $\Gamma$.

Remark 5.2. Similarly, if we take $s \triangleq e^{-\lambda \tau}=u_{1}=v_{1}=0$ in (3.15), then $x_{1}=y_{0}=u_{0}=v_{0}=u_{1}=$ $v_{1}=0$ (i.e., condition (5.1)), it means that there is a symmetric 1-homoclinic orbit to $O$ if and only if $s=u_{1}=v_{1}=0$ in Section 4 .

Remark 5.3. In Theorem 4.2 (resp., Theorem 5.1), the geometric meaning of $k$ is that, confined in $U_{0}$, the $u-v$ component of the above 1-periodic orbit bifurcated from $\Gamma$ makes circle $k$ (resp., $2 k$ ) times around the saddle-center.

Remark 5.4. From $x_{1} / \sqrt{s}=\delta \sqrt{s}(1+O(\delta)), s=O\left(\epsilon^{2}\right)$ and the constitution of $\rho_{11}$ and $\rho_{21}$, it is easy to know that the necessary condition for $\rho_{11}=\rho_{21}$ is $O_{1}\left(y_{1}\right)=O_{2}\left(y_{1}\right)$.

\section{Acknowledgments}

This paper is supported by NSF of China (11101370, 11126265, 11171085), the Research Fund for the Doctoral Program of Higher Education (20111420120006), and Zhejiang Provincial NSF of China (Y6100081).

\section{References}

[1] Y. L. Jin and D. M. Zhu, "Bifurcations of rough heteroclinic loop with two saddle points," Science in China A, vol. 46, no. 4, pp. 459-468, 2003.

[2] O. Yu. Koltsova, "Families of multi-round homoclinic and periodic orbits near a saddle-center equilibrium," Regular \& Chaotic Dynamics, vol. 8, no. 2, pp. 191-200, 2003.

[3] A. R. Champneys, "Homoclinic orbits in reversible systems and their applications in mechanics, fluids and optics," Physica D, vol. 112, no. 1-2, pp. 158-186, 1998.

[4] J. Klaus and J. Knobloch, "Bifurcation of homoclinic orbits to a saddle-center in reversible systems," International Journal of Bifurcation and Chaos in Applied Sciences and Engineering, vol. 13, no. 9, pp. 26032622, 2003.

[5] O. Yu. Koltsova and L. M. Lerman, "Periodic and homoclinic orbits in a two-parameter unfolding of a Hamiltonian system with a homoclinic orbit to a saddle-center," International Journal of Bifurcation and Chaos in Applied Sciences and Engineering, vol. 5, no. 2, pp. 397-408, 1995.

[6] X. B. Liu, X. L. Fu, and D. M. Zhu, "Bifurcation of homoclinic orbits with saddle-center equilibrium," Chinese Annals of Mathematics B, vol. 28, no. 1, pp. 81-92, 2007.

[7] A. Mielke, P. Holmes, and O. O'Reilly, "Cascades of homoclinic orbits to, and chaos near, a Hamiltonian saddle-center," Journal of Dynamics and Differential Equations, vol. 4, no. 1, pp. 95-126, 1992.

[8] K. Yagasaki and T. Wagenknecht, "Detection of symmetric homoclinic orbits to saddle-centres in reversible systems," Physica D, vol. 214, no. 2, pp. 169-181, 2006.

[9] D. M. Zhu, "Problems in homoclinic bifurcation with higher dimensions," Acta Mathematica Sinica, vol. 14, no. 3, pp. 341-352, 1998. 
[10] Y. C. Xu, D. M. Zhu, and F. J. Geng, "Codimension 3 heteroclinic bifurcations with orbit and inclination flips in reversible systems," International Journal of Bifurcation and Chaos in Applied Sciences and Engineering, vol. 18, no. 12, pp. 3689-3701, 2008. 


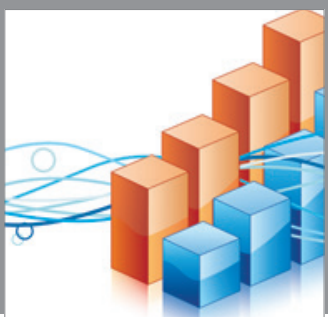

Advances in

Operations Research

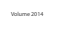

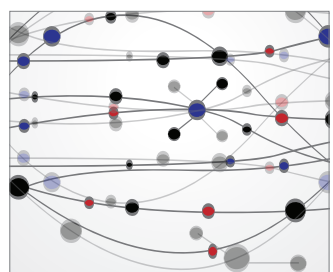

\section{The Scientific} World Journal
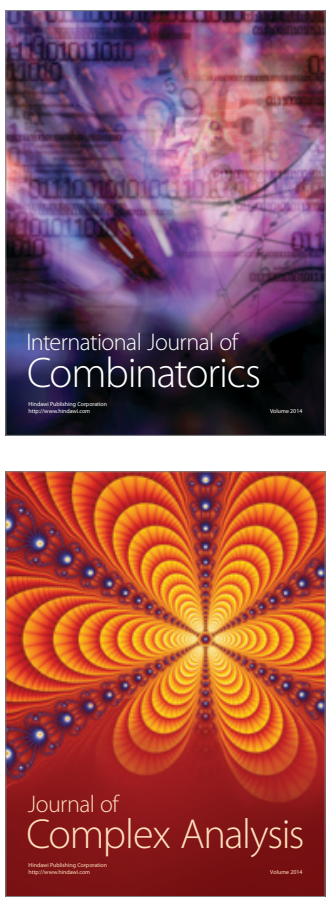

International Journal of

Mathematics and

Mathematical

Sciences
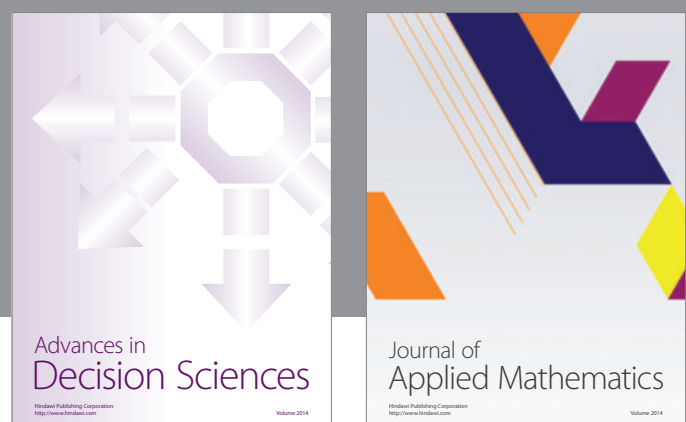

Journal of

Applied Mathematics
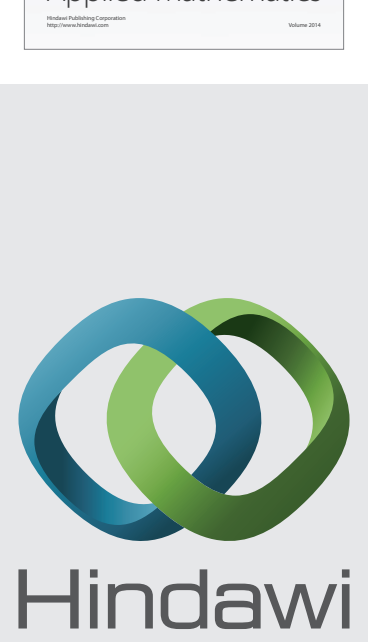

Submit your manuscripts at http://www.hindawi.com
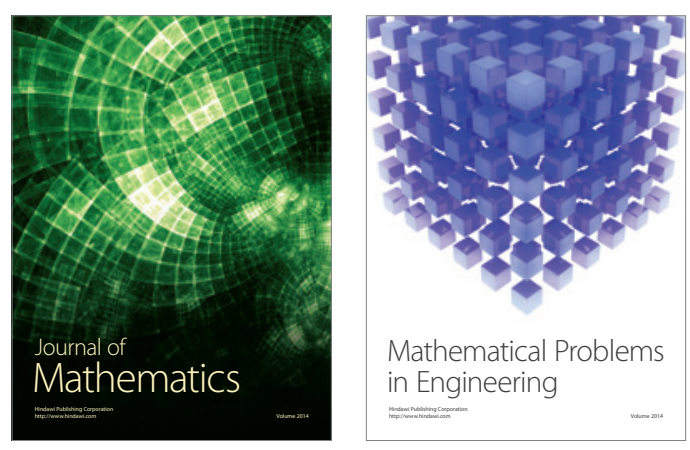

Mathematical Problems in Engineering
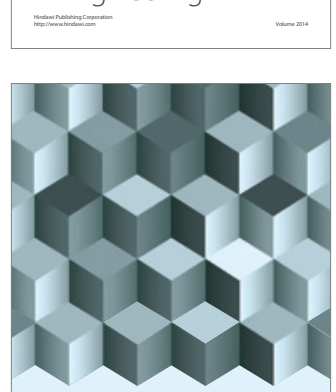

Journal of

Function Spaces
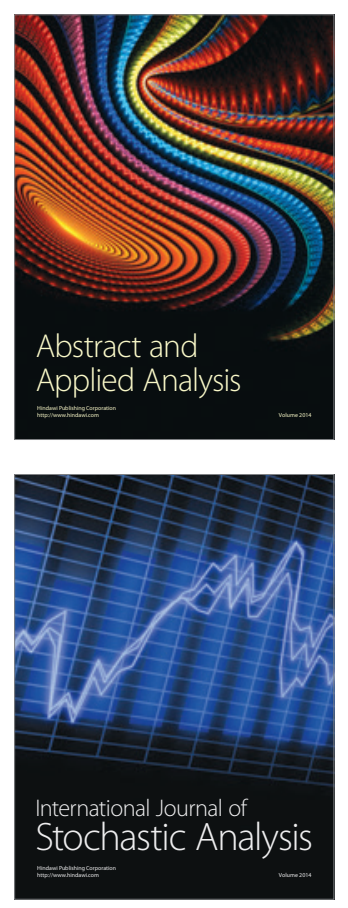

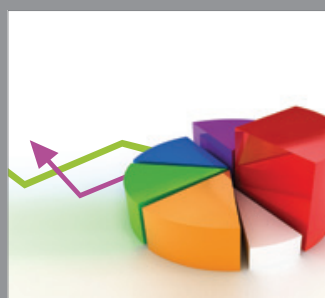

ournal of

Probability and Statistics

Promensencen
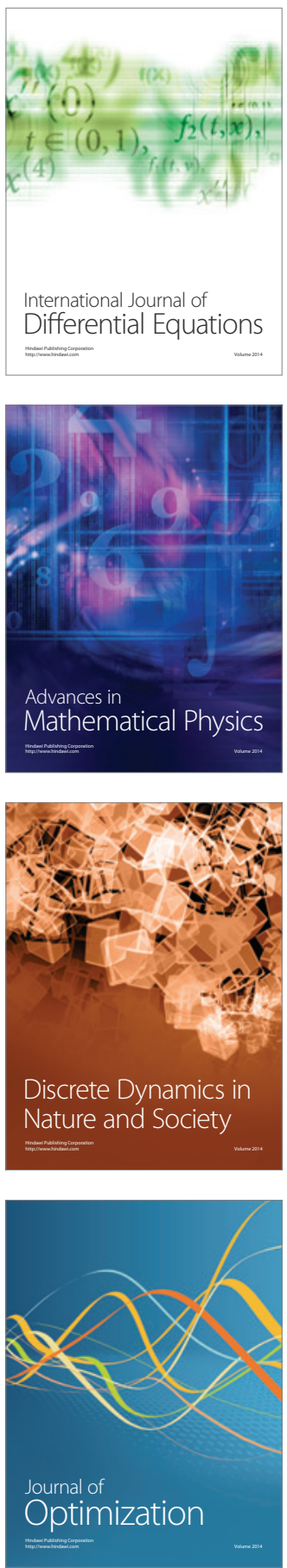